\title{
Short-term outcome of acute appendicitis at a rural surgical facility in Sri Lanka; a disease beyond benign simplicity
}

\author{
P. M. Jayawickrama ${ }^{1}$, J. A. S. B. Jayasundara ${ }^{1,2}$ \\ ${ }^{1}$ District General Hospital, Mannar, Sri Lanka \\ ${ }^{2}$ District General Hospital, Nuwaraeliya, Sri Lanka
}

Keywords: Acute appendicitis; appendicectomy; alvarado score

\section{Abstract \\ Introduction}

Acute appendicitis is the commonest intra-abdominal emergency requiring surgical intervention. The preferred treatment is appendicectomy with variable access techniques, but some patients with uncomplicated appendicitis respond well to antibiotics. Despite the benign nature of the disease, complications are not uncommon in appendicitis and after appendicectomy.

\section{Methods}

Outcome data of all cases diagnosed as acute appendicitis at District General Hospital, Mannar were prospectively collected from August 2016 to April 2017. Association between the age and duration of symptoms with the operative findings and postoperative complications were evaluated. Clavien-Dindo classification was used to grade the complications. Cases were reviewed at one month and one year.

\section{Results}

Out of 96 cases (Mean age 25.5 years, Range 7-64) diagnosed with acute appendicitis 43 were males. Eighty-three patients underwent surgery (Open appendicectomy-48, Laparoscopic appendicectomy-30, Appendicular abscess drainage-3, Midline laparotomy-2). Seventy-three (76\%) and twentythree $(24 \%)$ cases presented with less than two and more than three days of symptoms respectively. Fifty $(60.3 \%)$, twentyeight $(33.7 \%)$ and five $(6 \%)$ cases had uncomplicated appendicitis, complicated appendicitis and macroscopically normal appendix as operative findings. Mean age of 30.9 years in the cases with complicated appendicitis was significantly higher than the cases with uncomplicated appendicitis (23.7 years) or ones with normal-looking appendix (22.8 years) $(\mathrm{p}=0.007$ and $\mathrm{p}=0.004$, post-hoc ANOVA). Mean duration of symptoms of 2.5 days noted in

Correspondence: Bingumal Jayasundara

E-mail: bingumalj@gmail.com

(i) https://orcid.org/0000-0002-6435-0734

Received: 29-03-2020 Accepted: 13-08-2020

DOI: http://doi.org/10.4038/sljs.v38i2.8676 the cases with complicated appendicitis was significantly higher than the ones in the other groups (1.75 and 1.65 days) ( $\mathrm{p}<0.001$ and $\mathrm{p}<0.001$ post-hoc ANOVA). Eighteen $(21.7 \%)$, five $(6 \%)$ and one $(1.2 \%)$ cases had grade $1-2$, grade 3-4 and grade 5 complications on Clavien-Dindo classification respectively. Mean age of 46.8 years in the cases with grade 3-5 complications was significantly higher than 24.4 years in the cases with no complications or 22 years in the ones with lesser complications $(p=0.001$ and $p<0.001$ posthoc ANOVA). Mean duration of symptoms of cases with grade 3-5 complications (3.25 days) was higher than the rest. Sixteen of $28(57.14 \%)$ with complicated appendicitis developed postoperative complications.

\section{Conclusions}

Within the study cohort, advanced age and prolonged symptoms were risk factors for complicated appendicitis. Advanced age was associated with severity but not the occurrence of postoperative complications in acute appendicitis. Delayed presentation and complicated appendicitis lead to post-operative complications.

\section{Introduction}

Acute appendicitis is the commonest intra-abdominal inflammatory disease requiring surgical intervention worldwide [1, 2]. It is also the most frequent surgical emergency in childhood [2]. Appendicitis is widely seen between the ages of 10 to 20 years but there is no age exemption $[1,2]$. Lifetime risk ranges from 7 to $14 \%$ across both genders and females tend to have a slightly higher risk range [3]. In 1886, Dr Reginald Fitz emphasized that the vermiform appendix as the commonest culprit for the inflammation of the right iliac fossa $[1,4]$.

Since then several postulations have been made on the pathophysiology of acute appendicitis. Out of which the most popular theory being the luminal obstruction with a faecolith followed by distension of the appendix leading to bacterial overgrowth, raised intraluminal pressure with tissue compromise and gangrenous perforation as the result $[1,3]$. Even 125 years after the first documented diagnosis of appendicitis, the exact pathophysiology of this common disease is still unclear as solid supporting evidence is lacking for the theory of fecolith obstruction leading to luminal 
hypertension [3]. Appendicectomy remains the foremost mode of treatment in acute appendicitis [1,3]. Variable access techniques have been utilized by surgeons over the years, ranging from the midline or paramedian laparotomies used in the nineteenth century, famous right iliac fossa muscle splitting incision of McBurney preferred laparoscopic minimal access techniques of the late twentieth century to natural orifice transluminal surgery (NOTES) and endoluminal surgery in the twenty-first century $[1,4,5]$. Some patients with acute appendicitis respond well to antibiotics, a modality that has been successfully practised in naval and military medicine due to lack of access to the surgical facility [6]. There have been several trials to assess the efficacy of antibiotic therapy alone or antibiotic first policy in appendicitis with varied conclusions $[1,3,7,8$,$] .$

Several governing bodies in general or gastrointestinal surgery and emergency medicine still recommend appendicectomy as the treatment of choice for acute appendicitis and states antibiotic first policy 'may be effective with the potential risk of reoccurrence' [3]. Despite all the advances in medical knowledge and surgical technology of the twenty-first century, there were 54000 deaths caused by appendicitis around the globe in 2016 as estimated by the World Health Organization and almost two-thirds of such fatalities occurred in low-income countries in Africa and South-east Asia [9]. Most of these deaths happen due to complications of the disease with intra-abdominal sepsis. Although appendicectomy is considered a relatively safe procedure, surgery for uncomplicated appendicitis still carries a mortality rate of 0.8 per 1000 procedures [2].

Sri Lanka is a third world developing country in South Asia with a gross domestic product per capita of $\$ 4073$ in 2017 and utilizes less than $3 \%$ of gross domestic product for the health expenditure $[10,11]$. However, the country has a wellestablished state-driven free health care system with average health indices comparable to the developed world despite the limited health care budget. Mannar is one of the least developed districts of the country with a population of 106000. District General Hospital, Mannar is manned by two general surgeons with continuous specialists' cover despite limited resources and manpower.

The institution has limited critical care support and lacks a facility for computed tomography and specialist anaesthesiology and radiology cover over weekends. In such a background, this study attempts to audit the outcome of the cases with acute appendicitis managed at the index low resourced institution and to identify the risk factors for complicated disease and post-operative complications.

\section{Methods}

Outcome data of all cases diagnosed as Acute Appendicitis managed at District General Hospital, Mannar were prospectively collected for eight months between August 2016 and April 2017. The ultimate working diagnosis was made by one of the Consultant Surgeons upon clinical findings and basic haematology, biochemistry and urinalysis; occasionally supported by abdominal ultrasonography. Alvarado score was used as an aid for decision making [12]. Management decision on surgery or antibiotic therapy (CoAmoxiclav or Cefuroxime with Metronidazole) was taken by the surgeon upon the clinical picture without randomization. For the cases commenced on the trial of antibiotic therapy, failure to experience a reasonable clinical response at 48 hours was considered as an indication for operative management.

Access mode of surgery was decided by the same surgeon accordingly without randomization, to best suit the clinical scenario. Association between the age of the patients and the duration of symptoms with the operative findings and postoperative complications were correlated. Operative findings were classified as uncomplicated appendicitis, complicated appendicitis and negative appendicitis. Complicated appendicitis was defined as the presence of perforation, gangrenous appendix, and phlegmon or periappendiceal abscess formation.

Histopathological correlation was performed in all specimens and emphasis was made to confirm or exclude microscopic appendicitis in the cases with negative appendicitis. ClavienDindo classification was used to grade the complications. All patients were reviewed at one month and ones with complications or concerns according to the clinical needs subsequently. At one year from the index admission, cases were reviewed again or contacted over the phone to assess the outcome based on a structured questionnaire. Parametric tests were used for statistical evaluation when data followed a normal distribution.

\section{Results}

Ninety-six (49 males; age range 5-64 years, mean 20.8) patients were diagnosed as having acute appendicitis. Mean Alvarado score for cases diagnosed as having acute appendicitis was 7.2 (Range 5-10). Thirteen (6 males; age range 5-32 years, mean 16.4 ) were successfully managed with antibiotic therapy. Twelve out of them were adjudged as having uncomplicated appendicitis and the other had an appendicular mass confirmed by ultrasonography. Eightythree patients (43 males; age range 7-64 years, mean 25.5) underwent surgery which included three cases of 
unsuccessful antibiotic treatment. Mean age of cases successfully managed with antibiotics was significantly low ( $p=0.002$ Pooled t-test). Mean Alvarado score of the cases successfully managed with antibiotics was 5.9 and ones who had surgery as 7.4, and these values were statistically significant ( $\mathrm{p}=0.003$ Pooled t-test). Within surgical group sixty-three $(75.9 \%)$ cases presented within two days from onset of symptoms and rest presented with a delay of more than three days (Range 12 hours to 5 days). Among the 13 cases successfully managed with antibiotics ten presented within two days and the other three presented after five days from onset of symptoms (Range 6 hours to 7 days).

Attempted treatment with local remedies or other traditional medical modalities and socioeconomic reasons were the key reasons for delayed presentation. Surgical procedures performed included 48 Open appendicectomies with right iliac fossa incision, 30 Laparoscopic appendicectomies, three Open appendicular abscess drainages with lateral incisions and two Midline laparotomies. One laparotomy ended up with a Right hemicolectomy and during the other large peri appendiceal abscess was drained in addition to appendicectomy. On perioperative macroscopic appearance, 50 $(60.3 \%)$ had uncomplicated appendicitis and 28 (33.7\%) had complicated appendicitis. Five $(6 \%)$ cases underwent appendicectomy despite the gross appearance of the appendix is normal as no other structural cause was apparent to describe the symptoms.

Mean age of 30.9 years in the cases with complicated appendicitis was significantly higher than in the cases with uncomplicated appendicitis (23.7 years) or ones with normallooking appendix (22.8 years) $(\mathrm{p}=0.007$ and $\mathrm{p}=0.004$ posthoc ANOVA). Mean duration of symptoms of 2.5 days noted in the cases with complicated appendicitis was significantly higher than the ones in the other two groups mentioned above (1.75 and 1.65 days) ( $p<0.001$ and $p<0.001$ post-hoc ANOVA).

All three cases who had surgery due to a lack of response to antibiotic therapy had uncomplicated appendicitis. Eighteen (21.7\%) cases had Clavien-Dindo classification grade 1-2 complications and five (6\%) had grade 3-4 complications. There was one (1.2\%) mortality of a 64-year-old patient with ASA grade 3 presenting with five days of symptoms. Following laparotomy to drain a large peri appendiceal abscess patient went into multi-organ failure. The mean age of the cases ending up with postoperative complications was 24.7 years and of the others was 21.8 years. Mean age comparison between the two groups, cases with and without postoperative complications was not significant $(p=0.25$ Pooled t-test). Mean age of the cases with grade 3-5 complications was 46.8 years.
This was significantly higher than the mean age of 24.4 years in the cases without complications or mean age of 22 years in the ones with lesser complications $(p=0.001$ and $p<0.001$ post-hoc ANOVA). All five cases with apparently normal appendices had an uncomplicated recovery. Mean duration of symptoms was 1.9 days in the cases with no post-operative complications and 2.2 days for the ones with grade 1-2 complications. Mean duration of symptoms in cases with Clavien-Dindo grade 3-5 complications was 3.25 days and that was higher compared to the rest. Sixteen out of 28 patients with complicated appendicitis developed postoperative complications compared to 7 out of 50 cases with uncomplicated appendicitis.

All 78 cases with macroscopic appendicitis had histological confirmation of the disease. Out of the five negative appendicectomies two had transmural neutrophil infiltration confirming microscopic disease and the other three showed lymphoid aggregations. None of the specimens contained incidental neoplasia. All who underwent surgery except for the case of mortality were reviewed at one month, and 75 were available for the follow up at one year. Two cases had woundrelated concerns in one month. Seventy-five cases reviewed at one year included both and none had persistent surgical concerns. Nine out of 13 cases managed with antibiotics were contacted at one year. One of them had undergone laparoscopic appendicectomy during that time due to a subsequent acute attack. Another one has had a hospital admission for non-specific right iliac fossa symptoms and the rest were symptom-free.

\section{Discussion and conclusions}

Diagnosis of acute appendicitis may not be straight forward at times due to atypical presentation. Despite all technological advancements, clinical history and examination findings play a major role in the diagnosis [2]. There is no unique biomarker to confirm the diagnosis of appendicitis, but elevated white blood cell count with a left shift is the most used laboratory marker aided by elevated C- Reactive Protein levels [3]. Confirming appendicitis remains a challenge and scoring systems have been developed to aid identify the probability of having the disease. Alvarado score has been a useful adjunct for this matter since the description in $1986[3,12]$. Computed tomography and Magnetic Resonance Imaging have the highest specificity and sensitivity of the imaging modalities when compared with ultrasonography [3].

However, in settings with limited supporting facilities surgeons may still have to proceed solely upon clinical judgment on acute appendicitis. This audit shows that the clinical diagnosis of acute appendicitis can be made with reasonable accuracy with acceptable negative appendicectomy rates. 
Despite the developing trends to use antibiotics to treat uncomplicated acute appendicitis, both surgeons were keen on appendicectomy in most of the cases. A minority of cases with very early symptoms and another with mass formation were successfully managed without surgery. The mean age and Alvarado score of cases successfully managed with antibiotics were low. This fact suggests that the surgeons would feel safer to manage younger patients with 'less severe' clinical features of appendicitis with antibiotics, probably due to lesser possibility of missing a sinister pathology even without definitive imaging. Lack of imaging for confirmatory diagnosis of appendicitis may be considered as a limitation in the study.

Advanced age and delayed presentation with prolonged symptoms were risk factors for complicated appendicitis within the study cohort. Although it is not considered as a direct indicator of the severity of the disease, the Alvarado score as a predictor of the complicated nature of appendicitis during this study. Increased age was not associated with the occurrence of postoperative complications in acute appendicitis but was associated with the severity of the complications.

Delayed presentation and complicated appendicitis were risk factors for postoperative complications. Delay in seeking proper allopathic medical/surgical assistance by patients has always been a concern especially in developing countries and causative socioeconomic and cultural reasons are beyond a clinical discussion. Clinicians may not have direct control over these identified risk factors for complicated appendicitis or post-operative complications after appendicectomy. However, surgeons, especially in limited-resource setting, must be vigilant when dealing with cases of suspected appendicitis in older patients with comorbidities and ones presenting late to anticipate complicated disease and greater post-operative concerns. Such anticipation would help the surgeon to predict the 'uneasy' outcomes of common benign disease at presentation and allow space for detailed communication with the patient and family to minimize subsequent hardships.

\section{Presentation}

This study was presented at the Annual Scientific Session of the College of Surgeons of Sri Lanka and joint academic meeting with Royal College of Surgeons of Edinburgh in August 2017 at Kandy and won the award for the best outstation paper.

\section{Acknowledgement}

Authors would acknowledge Dr Buddhika Dassanayake, University Surgical Unit, Teaching Hospital, Peradeniya for his assistance with the statistical evaluation.
All authors disclose no conflict of interest. The study was conducted in accordance with the ethical standards of the relevant institutional or national ethics committee and the Helsinki Declaration of 1975, as revised in 2000 .

\section{References}

1. Wagner M, Tubre DJ, Asensio JA. Evolution and Current Trends in the Management of Acute Appendicitis. Surgical Clinics of North America 2018; 98: 1005-23. https://doi.org/10.1016/j.suc.2018.05.006

2. Humes DJ, Simpson J. Acute appendicitis. British Medical Journal 2006; 333: 530-4.

https://doi.org/10.1136/bmj.38940.664363.AE

3. Flum DR. Acute Appendicitis - Appendectomy or the "Antibiotics First" Strategy. New England Journal of Medicine 2015; 372: 1937-43.https://doi.org/10.1056/NEJMcp1215006

4. Fitz RH. Perforating inflammation of the vermiform appendix; with special reference to its early diagnosis and treatment. American Journal of Medical Sciences 1886; 92: 321-46.5. McBurney $\mathrm{C}$. The incision made in the abdominal wall in cases of appendicitis, with a description of a new method of operating. Annals of Surgery 1894; 20: 38-43.

https://doi.org/10.1097/00000658-189407000-00004

6. Wojciechowicz KH, Hoffkamp HJ, van Hulst RA. Conservative treatment of acute appendicitis: an overview. International Maritime Health 2010; 62: 265-72.

7. Varadhan KK, Neal KR, Lobo DN. Safety and efficacy of antibiotics compared with appendicectomy for treatment of uncomplicated acute appendicitis: meta-analysis of randomised controlled trials. British Medical Journal 2012; 344: e2156 https://doi.org/10.1136/bmj.e2156

8. Podda M, Cillara N, Di Saverio S Lai A, Feroci F, Luridiana G, Agresta F, Vettoretto N; ACOI (Italian Society of Hospital Surgeons) Study Group on Acute Appendicitis. Antibiotics first strategy for uncomplicated acute appendicitis in adults is associated with increased rates of peritonitis at surgery. A systematic review with meta-analysis of randomized controlled trials comparing appendectomy and non-operative management with antibiotics. Surgeon 2017; 15: 303-14. https://doi.org/10.1016/j.surge.2017.02.001

9. World Health Organization. Global burden of disease. Health statistics and information systems. Disease burden and mortality estimates: Cause-Specific Mortality, 2000-2016. https:/www.who.int/healthinfo/global_burden_disease/estimat es/en/Accessed and downloaded on 6th June 2019

10.The World Bank/Data/GDP per capita (current US\$) https://data.worldbank.org/indicator/ny.gdp.pcap.cd. Accessed on 25 th June 2019

11. World Health Organization. Health accounts. https://www.who.int/health-accounts/en/ Accessed on 25th June 2019

12. Alvarado A. A practical score for the early diagnosis of acute appendicitis. Annals of Emergency Medicine 1986; 15: 557-564. https://doi.org/10.1016/S0196-0644(86)80993-3 